\title{
EDITORIAL
}

\section{Healthy cognitive ageing with a healthier diet and better nutrition?}

\author{
European Journal of Clinical Nutrition (2014) 68, 1177-1178; \\ doi:10.1038/ejcn.2014.179
}

The growing epidemic of Alzheimer's disease (AD) and other forms of dementia concerns not only patients and their families, as well as scientists, but also health care providers and politicians worldwide. In 2010 it was estimated that 35.6 million people worldwide live with dementia. These numbers are expected to almost double every 20 years, thus, to 65.7 million by 2030 and 115.4 million by $2050 .^{1}$ Its consequences are not merely personal for the patients and their relatives but also socioeconomic. The estimated annual cost to society of dementia-US\$604 billion (roughly equivalent to $1 \%$ of world's GDP)-highlights the enormous impact that dementia has on socioeconomic conditions worldwide. ${ }^{2}$

Given the high individual and societal burden of dementia, as well as demographic changes (so-called 'population greying'), it is not surprising that a hunt for interventions against dementia became a scientific and health-care systems priority, particularly in the most affected regions of North America and Europe. The initiatives such as the EU Joint Programme - Neurodegenerative Disease Research (JPND), the European Innovation Partnership on Active and Healthy Ageing and Global Alliance for Chronic Diseases, and increased funding represent, not only better our understanding of what is important for population health worldwide but also give better opportunities to scientific collaboration and support novel interventional and preventive strategies against dementias and other chronic, mostly agerelated disorders.

Scientific progress has been made in terms of having a better understanding of the aetiology and pathogenesis of $A D$ and other dementias, however this improved knowledge has not yet translated into effective therapies. Moreover, treatment strategies believed to be strictly based on neurobiology of disease (such as active or passive immunotherapies or other $\beta$-amyloid-related interventions for $A D$ ) have so far been proven ineffective, scientific rationale (mostly the so-called amyloid cascade hypothesis) has been questioned and consequently, the interest of researchers moved from the dementia syndrome to preclinical, asymptomatic stages of $A D .^{3}$

On the other hand, some optimistic signals are coming from epidemiological studies.

First, long-term studies found and confirmed several potentially modifiable risk factors related to the development of $A D$ and other dementias. These include factors related to cardiovascular health interventions (such as hypertension, diabetes, atrial fibrillation and hypercholesterolaemia treatments) and lifestyle changes, including mostly dietary changes and regular (preferably aerobic) exercise as well as other less-studied factors (sleep routine maintaining, mental and societal activities). Identification of epidemiologic risk factors has recently led to the publication of preliminary expert-based guidelines. ${ }^{4}$

Second, recent prevalence and incidence estimates from North America and Europe showed that we might already see some decline in overall disease burden, which is attributed to lifestyle and health-related behaviour changes in these societies. ${ }^{5}$ Consequently, a panel of international experts has recently presented (during G8 summit in London earlier this year) a statement on dementia (including AD) prevention. The experts believe that there is already sufficient evidence to justify immediate action and call for not only preventive trials and healthy lifestyle changes promotion but also for an increase of government and international funding for research on the prevention of dementia. ${ }^{6}$

In this issue we provide a series of reviews on nutritional and fitness-related aspects of the dementias provided by the group of European experts that met during the Workshop 'Nutrition and Diet for Age-Related Cognitive Decline and Dementia' organised in Lodz, Poland (6-7 March 2014) within the framework of a multidisciplinary Healthy Ageing Research Centre (HARC; www.harc.umed.pl) established in the Medical University of Lodz and funded by EU-FP7 program. The papers discuss issues of cognitive decline diagnostics (Tsolaki; Bastin \& Salmon, this issue) and strongly support the idea of inclusion of nutritional (and general fitness) assessments as part of clinical workout for patients with cognitive impairments. Several possible models of assessment are discussed, including both simple, wellestablished tools (such as body mass index or questionnaires) as well as more advanced body composition analyses and their practical utility in this particular group of patients (Garcia-Ptacek et al.; Sobow et al.; Müller et al.; Camina Martin et al.; Buffa et al. this issue). An innovative research project based on the abovementioned elements, aimed for delineating the influence of metabolic changes and body composition analysis on cognitive dysfunction in healthy elderly and cognitively impaired is also presented (Magierski et al., this issue). Coupled with papers dealing with epigenetics (Dauncey, this issue) and possible nutritional interventions (Woodside et al., this issue), we provide here a wider look on both possible dementia prevention and patients' quality of life amelioration through dietary/nutritional intervention but also, more generally speaking, provoke questions about possible aging process and its modification (BarbergerGateau, this issue). We hope to provide here a forum for discussion on these unsatisfactorily explored issues related to human cognitive decline and possibly open new possibilities for collaborative work against the dementias, a real plague of the 21 st century.

\section{CONFLICT OF INTEREST}

The author declares no conflict of interest.

Department of Medical Psychology, Medical University of Lodz Sterlinga 5, Lodz, Poland

E-mail: tomasz.sobow@umed.lodz.pl

\section{REFERENCES}

1 Prince M, Bryce R, Albanese E, Wimo A, Ribeiro W, Ferri CP. The global prevalence of dementia: a systematic review and metaanalysis. Alzheimers Dement 2013; 9: 63-75.

2 Wimo A, Jönsson L, Bond J, Prince $M$, Winblad B. The worldwide economic impact of dementia 2010. Alzheimers Dement 2013; 9: 1-11. 
3 Golde TE, Schneider LS, Koo EH. Anti-a $\beta$ therapeutics in Alzheimer's disease: the need for a paradigm shift. Neuron 2011; 69: 203-213.

4 Barnard ND, Bush Al, Ceccarelli A, Cooper J, de Jager CA, Erickson KI et al. Dietary and lifestyle guidelines for the prevention of Alzheiemr's disease. Neurobiol Aging 2014; 35: S74-S78.
5 Langa KM. Is the Risk of Alzheimer's Disease Declining? A Review of Evidence From Around the World. 2014 Alzheimer's Association International Conference: Copenhagen, Denmark, 2014 12-17 July.

6 Smith AD, Yaffe K. Dementia (including Alzheimer's disease) can be prevented: statement supported by international experts. J Alzheimers Dis 2014; 38: 699-703. 